\title{
High Performance Hydroxide Zinc Carbonate Composite for Supercapacitors
}

\author{
Yu Zhao, Yubin Yang, Ruiming Ren* \\ Liaoning Provincial Key Laboratory of New Energy Battery, Dalian Jiaotong University, Dalian, China \\ Email: ${ }^{\star} y z h a o 119 @ 126 . c o m$
}

How to cite this paper: Zhao, Y., Yang, Y.B. and Ren, R.M. (2019) High Performance Hydroxide Zinc Carbonate Composite for Supercapacitors. Journal of Materials Science and Chemical Engineering, 7, 54-60.

https://doi.org/10.4236/msce.2019.711006

Received: November 1, 2019

Accepted: November 26, 2019

Published: November 29, 2019

Copyright $\odot 2019$ by author(s) and Scientific Research Publishing Inc. This work is licensed under the Creative Commons Attribution International License (CC BY 4.0).

http://creativecommons.org/licenses/by/4.0/

\begin{abstract}
Energy density and production cost for high-performance electrode materials are the main challenge for the capacitive storage technology. In this paper, novel Hydroxide Zinc Carbonate $\left(\mathrm{Zn}_{4} \mathrm{CO}_{3}(\mathrm{OH})_{6} \cdot \mathrm{H}_{2} \mathrm{O}, \mathrm{HZC}\right)$ catalyst layers, which are composed of irregular stagger arrangement nanosheets, have been synthesized successfully on foam $\mathrm{Ni}$ from inorganic precursors by a feasible in situ hydrothermal method. Measured by electrochemical tests as electrode materials for supercapacitors, the HZC@Ni foam show high specific capacitance (1329.2 F.g $\mathrm{g}^{-1}$ at $1 \mathrm{~A} \cdot \mathrm{g}^{-1}$ and $882.8 \mathrm{~F} \cdot \mathrm{g}^{-1}$ at $10 \mathrm{~A} \cdot \mathrm{g}^{-1}$, respectively). These results show that the HZC@Ni foam could be a potential electrode material for supercapacitors. These encouraging results make these low-cost and eco-friendly materials promising for energy storage application.
\end{abstract}

\section{Keywords}

Hydroxide Zinc Carbonate, In situ Hydrothermal Method, Supercapacitor, Specific Capacitance

\section{Introduction}

Electrochemical capacitors (EC) or supercapacitors are a well-established class of energy storage devices and have been the subject of intense interest due to their high specific power density and fast recharge capabilities. Depending upon the charge transfer mechanisms, supercapacitors are divided into electrical doublelayer capacitors (EDLCs) and pseudocapacitors [1] [2] [3]. In EDLCs, the charge storage mechanism is non-faradic and the charges are accumulated in the interface between the electrode and electrolytic solution, while faradic redox reactions take place at the electrode surface in pseudo-capacitors [2]. The fast and the reversible faradic process along with non-faradic electric double layer formation allow pseudocapacitors to store higher energy than that of EDLCs [2]. 
The performance of pseudocapacitors mainly relies on the properties of the electrode materials [2]. $\mathrm{RuO}_{2}$ has been considered as one of the best-suited electrode material because of its high quasi-metallic conductivity and high pseudocapacitance [1]. However, the high cost and toxicity of the compound have placed huge obstacles for its large scale production. Therefore, many researchers are looking for various inexpensive transition metal oxides and hydroxides with high energy density as alternative electrode materials, like $\mathrm{ZnO}, \mathrm{TiO}_{2}$, and $\mathrm{MnO}_{2}$ as electrode materials of pseudocapacitors [3] [4] [5]. Among them, Zn-based materials have drawn great attention because of the low cost and excellent electrochemical properties [6] [7]. For instance, $\mathrm{ZnO} /$ graphene nanocomposite exhibited a specific capacitance of 280 at $1 \mathrm{~A} \cdot \mathrm{g}^{-1}$ [6]. Therefore, $\mathrm{ZnO}$ has great potential as the electrode material of supercapacitors. Huang et al. [7] synthesized microspherical $\mathrm{ZnO}$ which exhibited high specific capacitances of $1017.5 \mathrm{~F} \cdot \mathrm{g}^{-1}$ at 5 $\mathrm{A} \cdot \mathrm{g}^{-1}$. However, organic precursors complicate the synthesis process. So it is a challenge to prepare $\mathrm{Zn}$-based materials with feasible methods and enhanced electrochemical performance.

Herein, in this paper, we synthesize Hydroxide Zinc Carbonate (HZC) nanosheets on foam Ni by a feasible in situ hydrothermal method and with inorganic precursors. Electrochemical tests show that the HZC@Ni foam exhibited excellent electrochemical performance.

\section{Experimental}

All the chemical reagents were purchased from Sinopharm Chemical Reagent Co. Ltd. (Shanghai, China), which were of analytical purity and used without any further purification. Foam Ni was purchased from Shenyang Research Institute of Nonferrous Metals Co. Ltd. (Shenyang, China).

Nanosheet Hydroxide Zinc Carbonate is directly grown on foam Ni by a hydrothermal synthesis. Typically, Ni foam $(10 \mathrm{~mm} \times 10 \mathrm{~mm} \times 1 \mathrm{~mm})$ is ultrasonically washed successively with $3 \mathrm{~mol} \cdot \mathrm{L}^{-1} \mathrm{HCl}$ solution, deionized water, and absolute ethanol in an ultrasound bath for $10 \mathrm{~min}$ each to remove $\mathrm{NiO}$ layer and residual organics on the surface. An aqueous solution was prepared and stirred thoroughly to form a clear solution with $\mathrm{Zn}\left(\mathrm{NO}_{3}\right)_{2} \cdot 6 \mathrm{H}_{2} \mathrm{O}$ as precursor, urea as the precipitator, trace $\mathrm{Al}\left(\mathrm{NO}_{3}\right)_{3} \cdot 9 \mathrm{H}_{2} \mathrm{O}$ as lamellar structure agents. A cleared $\mathrm{Ni}$ foam sheet is put into a $50 \mathrm{ml}$ Teflon-lined autoclave containing above homogeneous solution. The autoclave was sealed, maintained at $120^{\circ} \mathrm{C}$ for $4 \mathrm{~h}$, and then cooled to room temperature naturally. The final product was thoroughly washed with $\mathrm{H}_{2} \mathrm{O}$ and dried at $60^{\circ} \mathrm{C}$ for $8 \mathrm{~h}$. The mole ratio of urea/Zinc is 5 .

Powder X-ray diffraction (XRD) technique ( $\mathrm{Cu} \mathrm{Ka} \mathrm{PW1710,} \mathrm{Netherlands)}$ was employed to analyze the crystal structure and phase composition of the samples. The morphological studies of HZC nanosheets were performed using field emission scanning electronic microscope (FESEM, JEOLJEM-6360LV, Japan) and transmission electron microscopy (TEM, H-800, Japan). Fourier transform infrared spectrum spectral (FTIR, TJ270-30A, China) studies were 
carried out in the frequency region $400-4000 \mathrm{~cm}^{-1}$.

The HZC@foam Ni samples for supercapacitor application were preliminarily explored by constructing the cell involving three electrodes system in $6 \mathrm{~mol} \cdot \mathrm{L}^{-1}$ $\mathrm{KOH}$ solution. In three electrodes, prepared HZC@Ni foam was used as working electrode, $\mathrm{Hg} / \mathrm{HgO}$ and platium plate were used as the reference and counter electrodes respectively. The cyclic voltammetry (CV) curves were obtained at different scan rates of $5-100 \mathrm{mV} \cdot \mathrm{s}^{-1}$ using CHI660D electrochemical analyzer.

\section{Results and Discussion}

\subsection{Characterization of HZC}

The crystallographic structures of the prepared materials were estimated by X-ray diffraction measurement. XRD pattern of the product is presented in Figure 1. The product shows diffraction peaks at $12.8^{\circ}, 19.3^{\circ}, 34.4^{\circ}$, etc. corresponding to typical lattice planes of $\mathrm{Zn}_{4} \mathrm{CO}_{3}(\mathrm{OH})_{6} \cdot \mathrm{H}_{2} \mathrm{O}$ (JCPDS: 011-0287). The diffraction peaks of these planes are especially strong while other peaks have relatively weak intensities, indicating the growth of the crystal along a definite direction. It can be seen from Figure 1 that $\mathrm{Zn}_{4} \mathrm{CO}_{3}(\mathrm{OH})_{6} \cdot \mathrm{H}_{2} \mathrm{O}$ has been formed even after $1 \mathrm{~h}$ hydrothermal process at $120^{\circ} \mathrm{C}$. With the extension of hydrothermal reaction time, the peak strength of characteristic diffraction peaks of products slightly increases, indicating that the material can be synthesized easily with low energy consumption. And more importantly, the crystal structure and composition of products are stable which is critical to cycle stability.

The FTIR spectrum of the product is given in Figure 2, confirming the presence of $\mathrm{CO}_{3}^{2-}$ as well as water molecules intercalated in the interlayer. The absorption band around $3452 \mathrm{~cm}^{-1}$ corresponds to the $\mathrm{O}-\mathrm{H}$ stretching vibration of water molecules in the interlayer, $\mathrm{H}$-bonded hydroxyl group and possible Metal-OH stretching vibration. The band at $1350-1550 \mathrm{~cm}^{-1}$ assigned to O-C-O

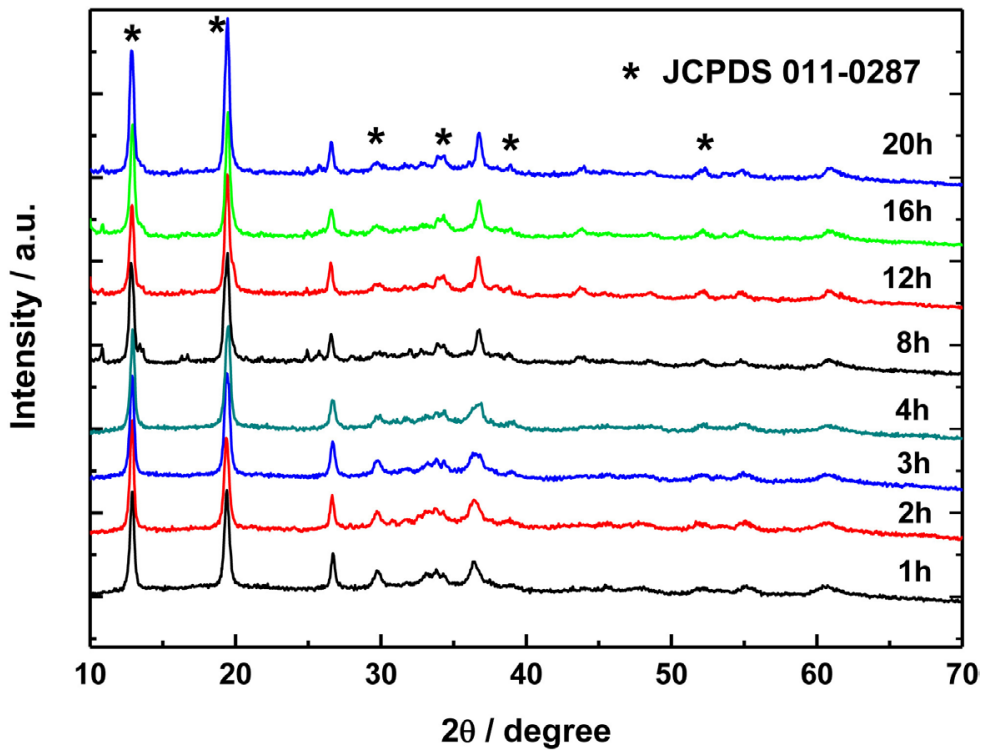

Figure 1. XRD pattern of as-synthesized HZC particles with different hydrothermal time. 
groups asymmetric vibration of single-base carbonate group ligand. Other absorption bands at $400-700 \mathrm{~cm}^{-1}$ are due to attributes to metal-oxygen (M-O) stretching and bending modes, including zinc-O-O, etc. All observations above are in good agreement with the previous reports [8] [9].

Hydrothermal technique over chemical routes is beneficial to control the morphology of products by properly choosing the temperature, time of reaction, solvent used for reaction, with or without the use of any major structure-directing agents and templates. The morphology of the prepared HZC growing on the surface of $\mathrm{Ni}$ foam is examined via field-emission scanning electron microscopy (FESEM). Figure 3(a) and Figure 3(b) show the morphological features of the HZC prepared in the present work at different magnifications. As presented in Figure 3(a), all of the HZC particles display nanosheets that are relatively loosely, irregularly grown on the substrate surface of foam Ni. The morphology of product particles is nearly hexagonal nanosheet with about $1-2 \mu \mathrm{m}$ of diameter and $50-100 \mathrm{~nm}$ thickness. The interconnected nanosheets form a mesoporous film on foam Ni surface.

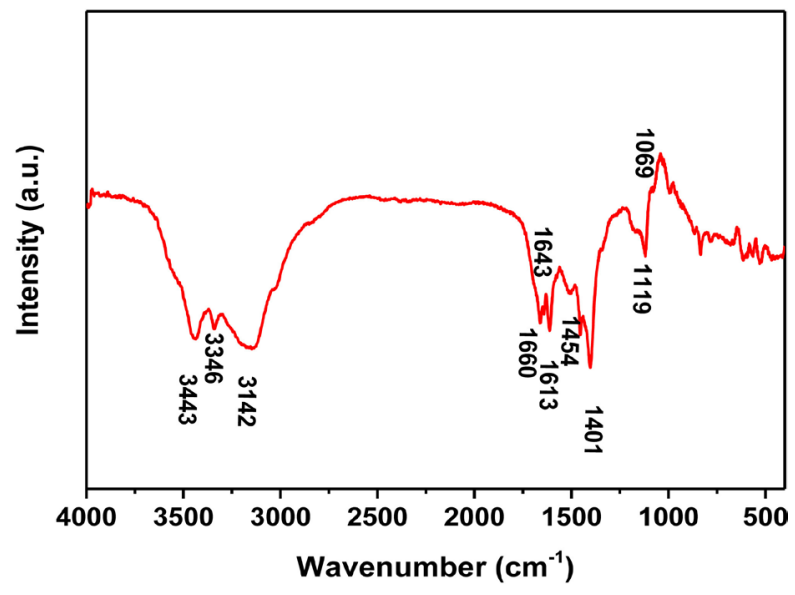

Figure 2. FTIR spectrum of as-synthesized HZC particles.

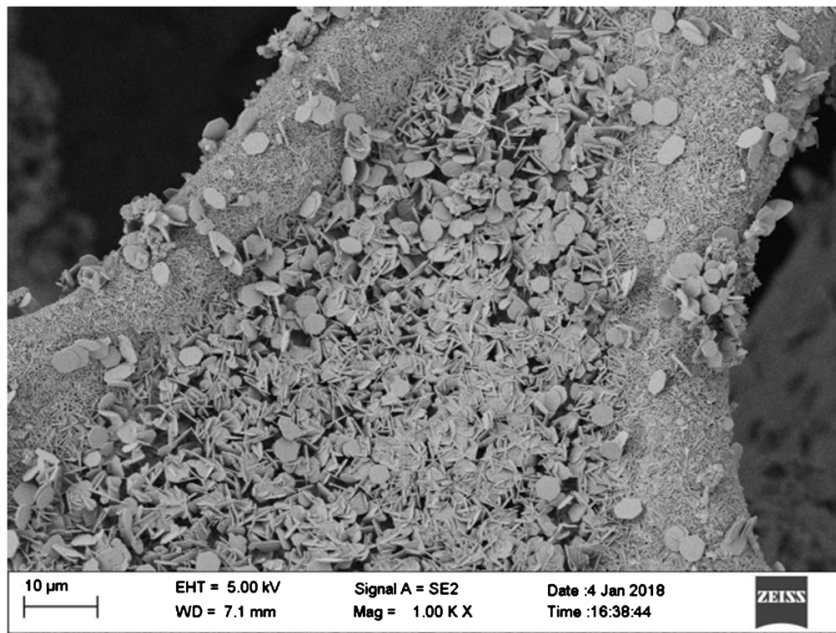

(a)

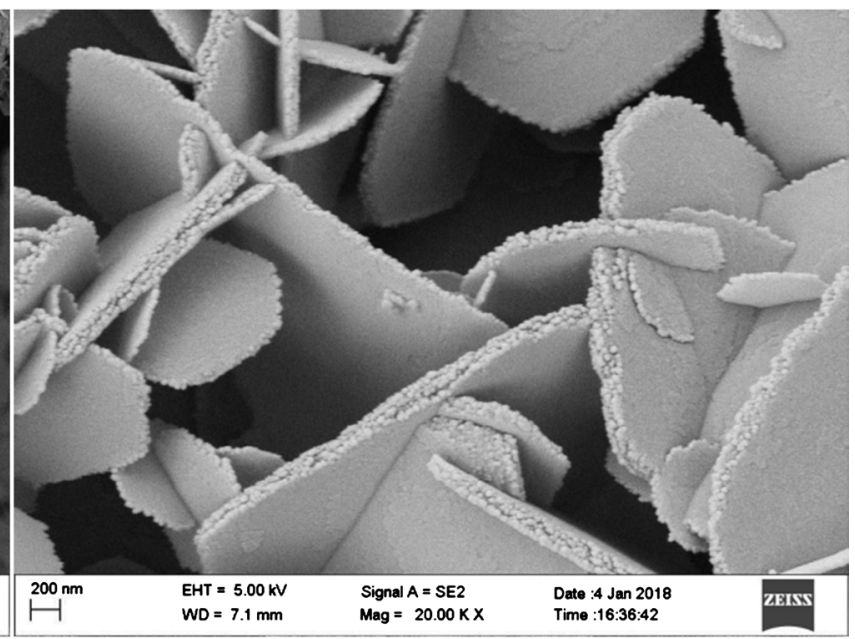

Figure 3. (a) SEM and (b) enlarged images of products. 


\subsection{Electrochemical Performance}

The electrochemical richness of HZC composite electrode has been extensively studied using cyclic voltammetry, galvanostatic charge-discharge studies and electrochemical impedance techniques. The CV traces (Figure 4) of HZC shows a pair of redox peaks in the range of $0-0.6 \mathrm{~V}$ (vs $\mathrm{Hg} / \mathrm{HgO}$ ) of $\mathrm{HZC}$ composite electrode in three electrode system, which indicates that HZC composite electrode exhibits typical energy storage mode of Faraday pseudocapacitance [3]. It is generally believed that the pseudocapacitance energy storage mechanism of $\mathrm{Zn}$ (II)-based oxide or hydroxide electrode in alkaline electrolyte solution is derived from the redox reaction of $\mathrm{Zn}(\mathrm{II})$, which is completed with the assistance of ion insertion and removal in electrolyte solution. Theoretical analysis shows that $E^{\theta}\left(\mathrm{Zn}^{2+} / \mathrm{Zn}\right)=-0.762 \mathrm{~V}$, so it can be inferred that $E^{\theta}\left(\mathrm{Zn}(\mathrm{OH})_{2} / \mathrm{Zn}\right)=-1.240$ $\mathrm{V}$. In the experiment, the redox peak of was about $0.45-0.5 \mathrm{~V}$ (vs $\mathrm{Hg} / \mathrm{HgO})$. Based on the literature, it was preliminarily speculated that the redox process of the active component of HZC electrode under the study conditions was between Zn (II) and Zn (III).

Galvanostatic charge-discharge curves (Figure 5) of HZC were recorded at different current densities in the potential range of $0-0.6 \mathrm{~V}$. From the constant-current charge and discharge curves, it can be seen that the curves are nearly symmetrical, with obvious charging and discharging platforms around $0.45 \mathrm{~V}$. The charging curves with relatively slow voltage change at the range of $0.45-0.6 \mathrm{~V}$ and the discharging curves at the range of $0.3-0.45 \mathrm{~V}$ both reflect the typical pseudocapacitive properties of HZC composite electrode, and correspond to the redox peaks in the CV curve of the electrode in Figure 4.

The specific capacitance of HZC composite electrode can be estimated from the discharge current using the following equation [3]:

$$
C=I \times t /(m \times \Delta V)
$$

where, $C\left(\mathrm{~F} \cdot \mathrm{g}^{-1}\right)$ is the specific capacitance of the electrode. $I(\mathrm{~A})$ is charge and

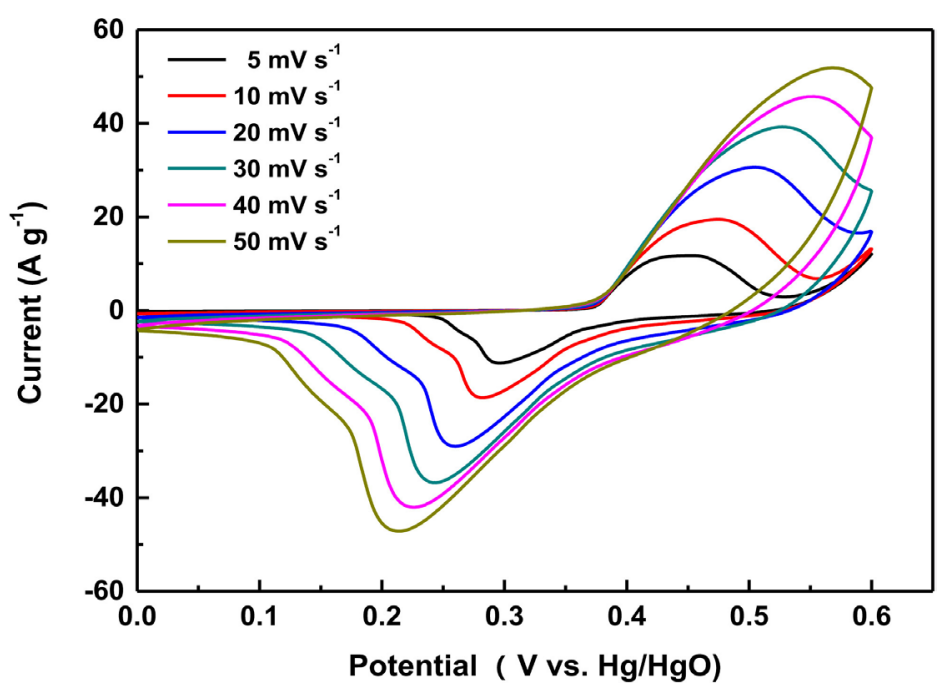

Figure 4. CV curves of electrode at different scan rate in $6 \mathrm{~mol} \cdot \mathrm{L}^{-1} \mathrm{KOH}$. 


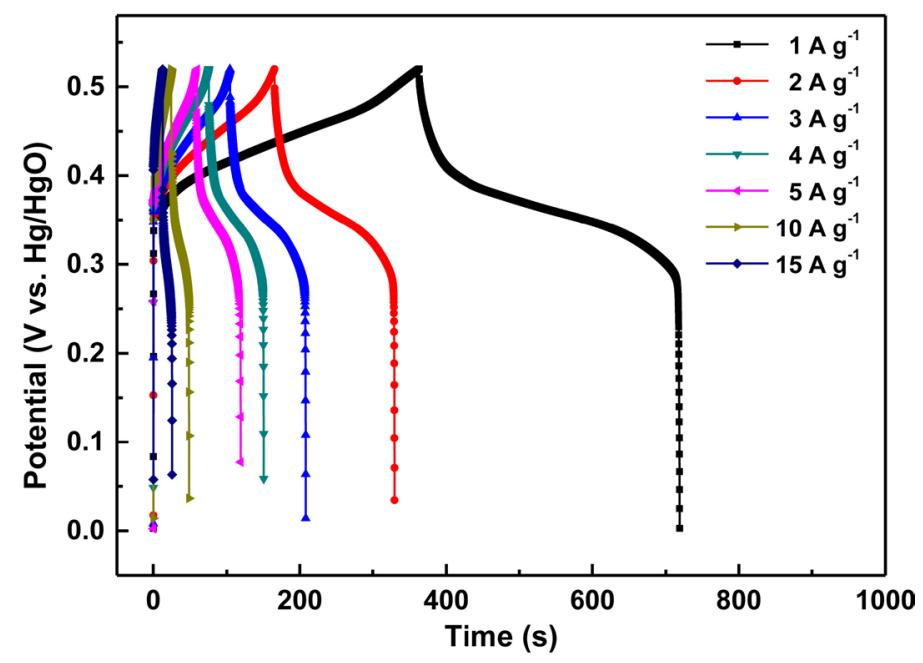

Figure 5. Galvanostatic charge and discharge curves of HZC electrode.

discharge current. $T(\mathrm{~s})$ is discharge time and $\Delta V(\mathrm{~V})$ for charging and discharging voltage window, $m(\mathrm{~g})$ as electroactive material quality. The estimated specific capacitance of HZC composite electrode is $1329.2 \mathrm{~F} \cdot \mathrm{g}^{-1}$ at a current density of $1 \mathrm{~A} \cdot \mathrm{g}^{-1}$ and $882.8 \mathrm{~F} \cdot \mathrm{g}^{-1}$ at $10 \mathrm{~A} \cdot \mathrm{g}^{-1}$, respectively. HZC in situ grown on foam Ni exhibits 40 fold enhancement in the capacity to store charges, when compared to HZC powders coated on foam Ni under the same test condition $\left(32.7 \mathrm{~F} \cdot \mathrm{g}^{-1}\right.$ at $\left.1 \mathrm{~A} \cdot \mathrm{g}^{-1}\right)$. By comparing the research progress and electrochemical performance results of $\mathrm{Zn}$-based electrode materials in recent years, it can be seen that a one-step hydrothermal process using inorganic precursors in this experiment is facile, and the synthesized HZC electrode materials show superior electrochemical activities [10] [11].

More advantageous crystal structure and the nanosheet morphology of HZC on foam $\mathrm{Ni}$ are the possible reasons for the excellent charge storage characteristics. Thin nanosheet arrays of uniform lamellar provide both large active surface area and good electrical conduction for fast redox kinetics. The layed structure of HZC can increase the rate of active centers participate in electrochemical reaction. This result shows that the ordered electrode material has a significant influence on the electrochemical performance of the HZC electrode. It is calculated that the energy density can reach $38.9 \mathrm{Wh} \cdot \mathrm{kg}^{-1}$ when the power density is $2000 \mathrm{~W}$.

\section{Conclusion}

$\mathrm{Zn}_{4} \mathrm{CO}_{3}(\mathrm{OH})_{6} \cdot \mathrm{H}_{2} \mathrm{O}$ composite electrode material was synthesized by a feasible in situ hydrothermal method with inorganic precursor. HZC particles on foam $\mathrm{Ni}$ were regular multi-layer nanosheets. The synthesized HZC electrode shows novel electrochemical activity in three electrode system. The specific capacitance of the electrode can reach about $1359 \mathrm{~F} \cdot \mathrm{g}^{-1}$ at the current density of $1 \mathrm{~A} \cdot \mathrm{g}^{-1}$. The next work will further refine the process of synthesis, analysis mechanism, and improve the cycle stability of the electrode materials. 


\section{Conflicts of Interest}

The authors declare no conflicts of interest regarding the publication of this paper.

\section{References}

[1] Hwang, J.Y., El-Kady, M.F., Wang, Y., Wang, L., Shao, Y., Marsh, K., Ko, J.M. and Kaner, R.B. (2015) Direct Preparation and Processing of Graphene/ $\mathrm{RuO}_{2}$ Nanocomposite Electrodes for High-Performance Capacitive Energy Storage. Nano Energy, 18, 57-70. https://doi.org/10.1016/j.nanoen.2015.09.009

[2] Lin, Z., Goikolea, E., Balducci. A., Naoi K., Tabeerna, P.L., Salanne. M., Yushin, G. and Simon, P. (2018) Materials for Supercapacitors: When Li-Ion Battery Power Is Not Enough. Materials Today, 21, 419-436. https://doi.org/10.1016/j.mattod.2018.01.035

[3] He, X., Yoo, J.E., Lee, M.H. and Bae, J. (2017) Morphology Engineering of ZnO Nanostructures for High Performance Supercapacitors: Enhanced Electrochemistry of $\mathrm{ZnO}$ Nanocones Compared to ZnO Nanowires. Nanotechnology, 28, 245402 245421. https://doi.org/10.1088/1361-6528/aa6bca

[4] Xu, Z., Sun, S., Cui, W., Yu, D. and Deng, J. (2018) Ultrafine $\mathrm{MnO}_{2}$ Nanowires Grown on RGO-Coated Carbon Cloth as a Binder-Free and Flexible Supercapacitor Electrode with High Performance. RSC Advances, 8, 38631-38640. https://doi.org/10.1039/C8RA05890C

[5] Zheng, L., Dong, Y., Bian, H., Lee, C., Lu, J. and Li, Y.Y. (2016) Self-Ordered Nanotubular $\mathrm{TiO}_{2}$ Multilayers for High-Performance Photocatalysts and Supercapacitors. Electrochimica Acta, 203, 257-264. https://doi.org/10.1016/j.electacta.2016.04.049

[6] Li, X., Wang, Z., Qiu, Y., Pan, Q. and Hu, P.A. (2015) 3D Graphene/ZnO Nanorods Composite Networks as Supercapacitor Electrodes. Journal of Alloys and Compounds, 620, 31-37. https://doi.org/10.1016/j.jallcom.2014.09.105

[7] Huang, G., Zhang, W., Xu, S., Li, Y. and Yang, Y. (2016) Microspherical ZnO Synthesized from a Metal-Organic Precursor for Supercapacitors. Ionics, 22, 2169-2174. https://doi.org/10.1007/s11581-016-1745-7

[8] Liu, Y. and Yang, Z. (2016) Intercalation of Sulfate Anions into a Zn-Al Layered Double Hydroxide: Their Synthesis and Application in Zn-Ni Secondary Batteries. RSC Advances, 6, 68584-68591. https://pubs.rsc.org/en/content/articlelanding/2016/ra/c6ra09096f https://doi.org/10.1039/C6RA09096F

[9] Peng, W., Li, H. and Song, S. (2017) Synthesis of Fluorinated Graphene/CoAlLayered Double Hydroxide Composites as Electrode Materials for Supercapacitors. ACS Applied Materials \& Interfaces, 9, 5204-5212. https://pubs.acs.org/doi/10.1021/acsami.6b11316 https://doi.org/10.1021/acsami.6b11316

[10] Luo, Q., Xu, P., Qiu, Y., Cheng, Z., Chang, X. and Fan H. (2017) Synthesis of ZnO Tetrapods for High-Performance Supercapacitor Applications. Materials Letters, 198, 192-195. https://doi.org/10.1016/j.matlet.2017.04.032

[11] Alver, Ü., Tanriverdi, A. and Akgül Ö. (2016) Hydrothermal Preparation of ZnO Electrodes Synthesized from Different Precursors for Electrochemical Supercapacitors. Synthetic Metals, 211, 30-34. https://doi.org/10.1016/j.synthmet.2015.11.008 